\title{
TRATAMENTO DE LESÕES DE MANCHA BRANCA ATIVA APÓS TRATAMENTO ORTODÔNTICO: RELATO DE CASO CLÍNICO
}

\author{
*Elaine Cristina Velêz Rodrigues I \\ Erika dos Santos Oliveira Trindade " \\ Flávia Roberta Costa de Azevêdo " \\ Nádja Inglide Lima de Brito Teixeira II \\ Suelen Farias Costa dos Santos " \\ Jainara Maria Soares Ferreira"
}

\section{RESUMO}

As lesões de mancha branca são a primeira expressão clínica da desmineralização que ocorre na superfície do esmalte do dente. Nesse sentido, este trabalho objetiva relatar um caso clínico sobre tratamento de lesões de mancha branca ativas em paciente de 19 anos, tratada ortodonticamente por 2 anos. Após remoção do aparelho, verificou-se presença de manchas brancas ativas nos elementos dentários permanentes $(n=10)$, as quais foram avaliadas clinicamente em relação a atividade (textura e luminosidade e a dimensão (maior diâmetro cérvico-incisal e mésio-distal) e os dados anotados no prontuário da paciente. Os dados foram analisados num enfoque quantitativo e qualitativo, sendo confrontados com a literatura pertinente ao tema. Após orientação de higiene e dieta, 8 sessões de fluorterapia com o verniz fluoretado Duoflorid XII® da FGM (6\% de fluoreto de cálcio e 6 \% de fluoreto de sódio) com profilaxia prévia usando pasta profilática SHINE ${ }^{\circledR}$ da Maquira (2000 ppm de F), foram observados os elementos redução das médias de diâmetros cervico-oclusal e médio-distal finais em 08 elementos e manutenção das médias em 02 . Além disso, 02 manchas brancas permaneceram ativas, as demais inativas. Conclui-se que houve remineralização das manchas brancas com redução do diâmetro e/ou atividade, após 8 sessões de terapia com verniz fluoretado e orientação quanto a higiene e dieta da paciente.

PALAVRAS-CHAVE: Cárie Dentária. Desmineralização. Flúor.

Doutora em Odontopediatria pela UPE. Docente do Curso de Odontologia da Faculdade Nova I Esperança, FACENE. Departamento de Odontologia. CEP 58067695, João Pessoa, Paraíba, Brasil.

*Autor correspondente: jainara.sf@gmail.com

Orcid: : 0000-0001-7040-7197

Dicente do Curso de Odontologia da Faculdade Nova Esperança, FACENE. Departamento de

Odontologia. CEP 58067695, João Pessoa, Paraíba, Brasil.

Orcid: 0000-0002-6549-2996; 0000-0002-2475-4734; 0000-0002-0193-314X; 0000-0001-9522-2754; 0000-0001-9374-1358 


\section{INTRODUÇÃO}

A cárie é uma das doenças bucais que mais acomete os brasileiros, apesar de existirem tratamentos eficazes para combatêla. ${ }^{1}$ As lesões de mancha branca são a primeira expressão clínica da desmineralização que ocorre na superfície do esmalte. ${ }^{2}$ Apresenta-se como uma opacidade localizada no esmalte, após os eventos físico-químicos ocorridos entre o esmalte e os ácidos produzidos pelas bactérias. $^{3}$

A perda mineral e as microporosidades são resultados desse processo e essas, por sua vez, podem ser reconhecidas clinicamente pela formação de áreas brancas, opacas e rugosas, podendo progredir, para lesões cavitadas se não tratadas. Quando detectada em sua fase inicial, ainda é passível de remineralização. ${ }^{4}$

Muitos pacientes com aparelho ortodôntico que não conseguem manter uma boa higiene oral, sobretudo aqueles que utilizam o aparelho fixo, apresentam lesões com manchas brancas. A dificuldade na limpeza dos dentes facilita o acúmulo de placa em volta bráquetes, bandas e fios ortodônticos elementos que podem favorecer a retenção de biofilme ${ }^{5}$ causando a desmineralização do esmalte e desencadeando as denominadas lesões de mancha branca. ${ }^{6}$ As lesões de manchas brancas são classificadas como ativas (ásperas e opacas) ou inativas (lisas e brilhantes). ${ }^{7,8,9}$ As lesões de mancha branca, causadas pela desmineralização do esmalte, são uma sequela comum e indesejável do tratamento ortodôntico, embora a prevenção destas lesões seja um dos objetivos do ortodontista. ${ }^{10,11,12}$
Apesar da cárie dentária ser descrita como um processo crônico que progride lentamente, sua progressão em pacientes com aparelho ortodôntico parece ser mais frequente. ${ }^{12,13} \mathrm{~A}$ prevalência de mancha branca em pacientes ortodônticos varia entre 2 e $97 \%$, detectadas frequentemente na região cervical dos dentes, adjacentes ao bráquete. ${ }^{14}$

O tratamento conservador de manchas brancas usando terapias remineralizadoras é um tema de interesse crescente entre clínicos e pesquisadores. ${ }^{15}$ Manchas brancas ativas podem ser paralisadas por meio do uso de produtos fluoretados, através da formação de fluorapatita e funcionado como reservatório de $\mathrm{CaF}_{2}$ liberando o íon para o meio bucal interferir com o processo de cárie. ${ }^{16,17,18,19}$

O flúor pode ser considerado como a substância mais utilizada na prevenção e/ou tratamento inicial da cárie dentária em todo o mundo e seu uso se dá de várias formas: água de abastecimento público fluoretada, dentifrícios, géis, vernizes, soluções e materiais dentários. ${ }^{20}$

A utilização de doses elevadas de fluoreto de forma sistemática por meio de fluorterapia tópica profissional tem sido sugerida durante e após o tratamento ortodôntico para áreas de desmineralização e prevenção da progressão de manchas brancas ativas de cárie. $5,11,12,13$

Desta forma, este trabalho objetiva relatar um caso clínico sobre tratamento de lesões ativas de mancha branca em paciente, tratada ortodonticamente, que buscou atendimento em consultório odontológico privado na cidade de João Pessoa-PB. 


\section{MATERIAL E MÉTODOS}

Este relato de caso envolveu uma paciente de 19 anos, que buscou atendimento em consultório odontológico privado em João Pessoa-PB. A paciente esteve sob tratamento ortodôntico fixo, com duração de 2 anos e foi examinada após este tratamento.

As lesões de mancha branca foram avaliadas por meio da medição com sonda WHO (TRINITY $\left.{ }^{\circledR}\right)$ e régua milimetrada (JON®) da média dos maiores diâmetros mésio-distal e cérvico-incisal, além da observação visual da atividade (textura e luminosidade), sendo consideradas manchas brancas ativas, aquelas rugosas e opocas e inativas, as lias e brilhantes. Previamente ao exame, foi realizada profilaxia prévia com pasta profilática SHINE ${ }^{\circledR}$ da Maquira (2000 ppm de F).

As lesões de mancha branca receberam 8 sessões semanais de fluorterapia com o verniz fluoretado DUOFLORID XII® da FGM (6\% de fluoreto de cálcio e $6 \%$ de fluoreto de sódio)

\section{RELATO DE CASO CLÍNICO}

Paciente de 19 anos, gênero feminino, usou por 2 anos aparelho fixo ortodôntico, classe ll com apinhamento inferior. Previamente ao referido tratamento, a paciente possuía $C P O-D=0$ e durante 0 referido tratamento, fazia manutenção ortodôntica mensalmente, momento em que realizava profilaxia profissional. Adotava uma dieta cariogênica (rica em carboidratos fermentáveis) e a higienização deficiente (uso eventual de fio dental e escovação dentária diária pouco frequente com dentifrício com 1450 ppm de $\mathrm{F}$ da Oral B). Durante o tratamento ortodôntico por meio de CAVIBRUSH® da FGM, seguindo recomendações de uso do fabricante.

Este estudo foi aprovado pelo Comitê de Ética em Pesquisa local (CAAE 34313520.8.0000.5184) e utilizou-se os pressupostos da Resolução 466/2012 CNS que trata de pesquisas e testes em seres humanos, como também do Código de Ética Odontológico.

Como instrumento de coleta de dados, utilizou-se o prontuário da paciente, sendo anotados os registros na evolução do paciente. Os dados foram analisados num enfoque quantitativo e qualitativo, sendo confrontados com a literatura pertinente ao tema, as quais foram incluídas neste estudo, através de realização de pesquisa em base de dados. com palavras chaves como, por exemplo: cárie dentária, desmineralização, esmalte dentário, fluorterapia e mancha branca.

não foi realizado o tratamento periodontal na paciente.

Após remoção do aparelho, verificouse presença de manchas brancas ativas nas vestibulares dos elementos 11, 12, 15, 21, 22, 24, 33, 34, 35 e 43, com médias de diâmetros cérvico-oclusal e médio-distal iniciais de, respectivamente, $5 \mathrm{~mm}, 4 \mathrm{~mm}, 2 \mathrm{~mm}, 3,5$ $\mathrm{mm}, 4 \mathrm{~mm}, 3 \mathrm{~mm}, 4 \mathrm{~mm}, 4 \mathrm{~mm}, 2 \mathrm{~mm}, 4$ $\mathrm{mm}$. Adicionalmente foi verificado presença de lesões cariosas com cavitação nas faces vestibulares elementos 13, 14, 23, 44, 45 $(C P O-D=5)$ (Figura 1). 


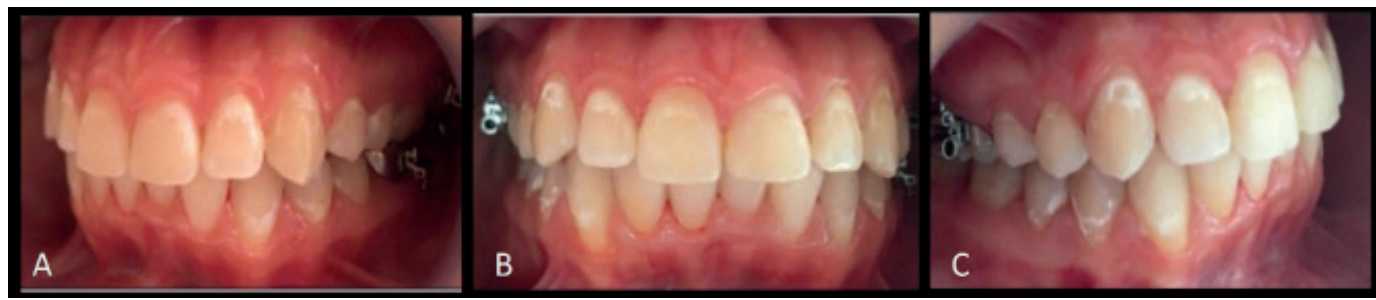

FIGURA 1: Aspecto Clínico Inicial da paciente após a remoção do aparelho fixo ortodôntico Lateral Esquerda (A), Frontal (B) e Lateral Direita (C).

Foi avaliada clinicamente a atividade (textura e luminosidade) e a dimensão (maior diâmetro cérvico-incisal e mésiodistal) das manchas brancas por meio de exame visual com sonda milimetrada WHO, respectivamente (Figura 2). Previamente a esta etapa, a superfície dentária recebia profilaxia prévia com pasta profilática SHINE ${ }^{\circledR}$ da Maquira (2000 ppm de F), além de secagem e iluminação adequadas.

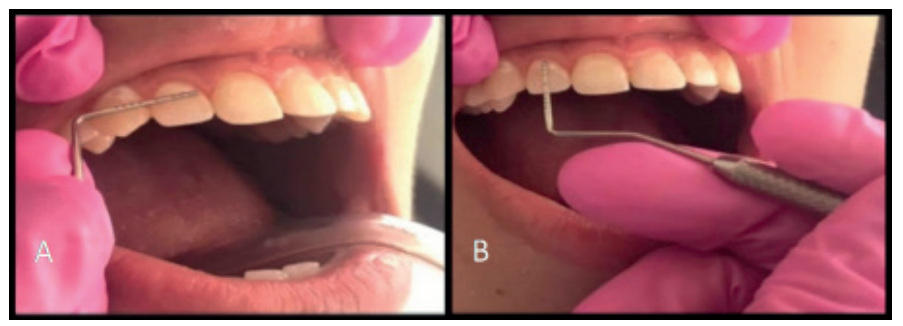

FIGURA 2: Medição do maior diâmetro M-D (A) e C-I (B) da mancha branca na paciente após a remoção do aparelho fixo ortodôntico.

Após orientação de higiene (controle mecânico do biofilme por meio de uso de escova de dente, dentrifício acima de 1000 ppm de flúor e fio dental) e dieta (diminuição de quantidade e frequência de carboidratos fermentáveis) e 8 sessões semanais de fluorterapia com o verniz fluoretado DUOFLORID XII® da FGM (6\% de fluoreto de cálcio e $6 \%$ de fluoreto de sódio), seguindo recomendações de uso do fabricante (Figura ${ }^{3}$ ), foram observados os elementos 11, 12, 15, 21, 22, 24, 33, 34, 35 e 43, com médias de diâmetros cérvico-oclusal e médio-distal finais de 3,$5 ; 3,5 ; 2 ; 3,5 ; 4 ; 2 ; 2 ; 3,5 ; 1,5 ; 3,5 \mathrm{~mm}$.

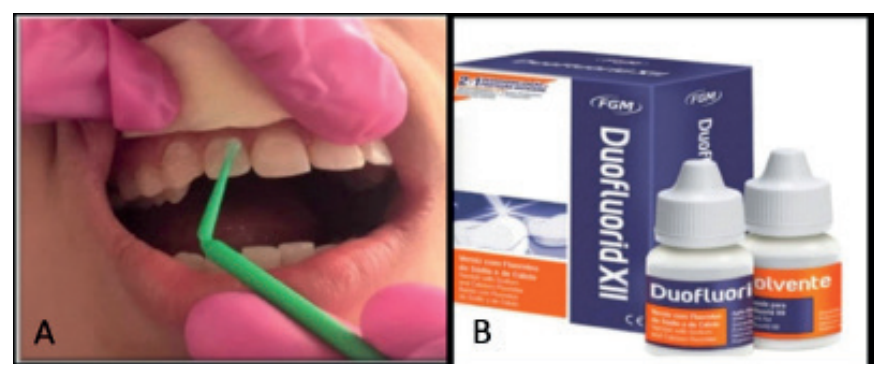

FIGURA 3: Realização de fluorterapia (A) conforme recomendação do fabricante usando o verniz fluoretado DUOFLORID XII® (B), na paciente após a remoção do aparelho fixo ortodôntico.

As manchas brancas dos elementos dentários 12 e 22 permaneceram ativas, as demais inativas. Adicionalmente, os elementos $13,14,23,44,45$ foram restaurados em resina 
composta com resina $A 2 E Z 350{ }^{\circledR}$ da 3 M com mínima intervenção nos tecidos dentários. Conclui-se que houve remineralização das manchas brancas com redução do diâmetro e/ ou atividade delas, após 8 sessões de terapia com verniz fluoretado e orientação quanto a higiene e dieta da paciente (Figura 4).

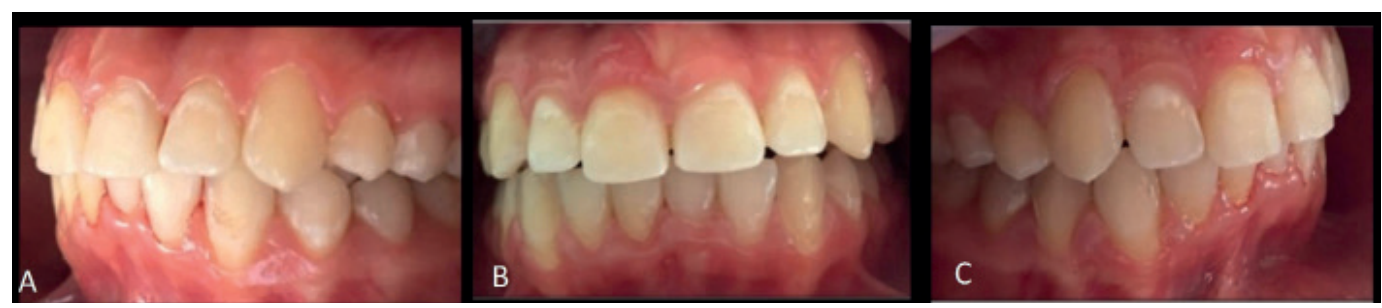

FIGURA 4: Aspecto Clínico Final da paciente Lateral Esquerda (A), Frontal (B) e Lateral Direita (C) paciente após realização de fluorterapia.

\section{DISCUSSÃO}

As manchas brancas podem ser uma sequela comum e indesejável do tratamento ortodôntico, principalmente com o uso prolongado, se caracterizando como um fator de risco quando atrelada a dieta cariogênica e a dificuldade de higienização, como no caso relatado, configurando fator importante na etiologia do aparecimento e progressão da lesão cariosa. ${ }^{21,22,23,24,25}$

A cárie dentária se caracteriza como um dos principais problemas de saúde bucal mais prevalentes no mundo ${ }^{3,24} \mathrm{e}$, atualmente, novos conceitos de classificação, diagnóstico e tratamento da doença permitem sua melhor compreensão, enfocando opções de tratamento minimamente invasivo, a exemplo fluorterapia. $., 10,14$

No caso clínico em questão, após tratamento ortodôntico de 02 anos, a paciente adquiriu 10 lesões de manchas brancas ativas e 05 lesões cariosas cavitadas, o que pode ser justificado pela deficiência de higiene bucal, especialmente na região dos dispositivos ortodônticos, devido ao acúmulo de biofilme dental nesta região, por serem retentores adicionais de biofilme dental. ${ }^{14}$

Esta situação pode ainda ser agravada devido a dieta cariogênica rica em carboidratos fermentáveis, ${ }^{21,22,23,24}$ como relatado pela paciente, caracterizando risco elevado para a doença cárie. Este tipo de lesão pode ser remineralizada através da utilização sistemática de produtos fluoretados na forma de verniz, espuma ou gel, além do controle mecânico criterioso do biofilme por meio da escovação diária dos dentes. ${ }^{26}$

Para o tratamento do caso clínico em tela, podemos contar com o tratamento de fluorterapia que tem como o principal mecanismo de ação do fluoreto a redução da velocidade com que as lesões de cárie progridem em um determinado período. ${ }^{27}$

Diante das diversas formas de apresentação de fluoretos, selecionamos para o caso em tela o verniz fluoretado, por possuir aderência a superfície dentária proporciona maior tempo de exposição dos fluoretos, segurança e facilidade de aplicação. ${ }^{22,24,25,27,28}$

$\mathrm{O}$ produto utilizado demonstrou eficácia na remineralização de dentes com eficácia na remineralização de dentes com manchas brancas, em relação as mudanças na atividade e no tamanho das manchas brancas. Adicionalmente, estudos prévios não apontam 
diferença estatisticamente significante com relação a sua eficácia clínica do DUOFLUORID XII® $(F G M)$ e de produtos importados, a exemplo do DURAPHAT ${ }^{\circledR}$ (COLGATE). ${ }^{23,24,25}$ Além disso, o verniz nacional é menos oneroso que o verniz importado, fator importante para a tomada de decisão de seleção, quer seja no serviço público, ou privado..$^{22,23,24,25}$

Quanto a frequência de aplicação dos vernizes fluoretados, optou-se por 8 sessões semanais, baseado na literatura comprobatória da obtenção de resultados eficazes ao submeterem lesões incipientes de cárie ativas a terapia com diferentes fluoretos, durante o mesmo período de aplicação. ${ }^{23,24,25}$

Para o caso em questão, foi recomendada a restrição temporária (12 horas) da escovação dentária aos pacientes da pesquisa, após a aplicação do DUOFLUORID $\mathrm{XII}$ (FGM), conforme recomendação do fabricante, com o objetivo de prolongar o tempo de adesão deste produto a superfície adamantina, maximizando assim seu efeito terapêutico.

Das 10 lesões ativas de mancha branca, ao final do tratamento, com relação a dimensão das lesões, 07 lesões diminuíram de tamanho e em 03 delas não houve alteração. No que concerne a atividade, 08 tornaram-se inativas e 02 ativas.

Neste sentido, a fluorterapia mostrouse eficaz na remineralização das lesões de manchabranca, umavezquehouvereduçãodos valores atribuídos aos métodos de diagnóstico do caso relatado. Estudos corroboram com nossos resultados de que o uso de fluoretos para o tratamento das alterações minerais dos dentes, provocadas pela doença cárie (fluorterapia), é uma estratégia comprovada para tal fim. ${ }^{15,29,23,24,25,28,30} \mathrm{Em}$ adição, o processo de remineralização destas lesões foi coadjuvado pelo uso do dentifrício fluoretado, durante a higienização bucal do paciente, e ainda pelo uso da pasta profilática mediante a profilaxia profissional.

É válido lembrar que as 05 lesões cavitadas de cárie foram restauradas de forma conservadora com resina composta, seguindo a filosofia da mínima intervenção aos tecidos dentários. ${ }^{30,31}$

\section{CONCLUSÃO}

A partir do caso relatado, podemos concluir a efetividade clínica do uso do verniz fluoretado em lesões iniciais de cárie, devido à redução de tamanho e inatividade da maior parte delas, após 8 sessões de fluorterapia em paciente tratado ortodonticamente, em comunhão com a aquisição de hábitos de higiene e dieta saudáveis.

\section{TREATMENT OF ACTIVE WHITE SPOT LESIONS AFTER ORTHODONTIC TREATMENT: CLINICAL CASE REPORT}

\section{ABSTRACT}

This paper aims to report a clinical case on the treatment of active white spot lesions in a 19-year-old 
patient treated orthodontically for 2 years. After removal of the braces, active white spots were found on permanent dental elements $(n=10)$, which were clinically evaluated for activity (texture and luminosity and dimension (larger cervical-incisal and mesio-distal diameter) and the data recorded in the patient's chart. The data were analyzed in a quantitative and qualitative approach, being compared with the relevant literature. After hygiene and diet guidance, 8 fluorotherapy sessions with the fluoride varnish Duoflorid XII® from FGM ( $6 \%$ of calcium fluoride and $6 \%$ of sodium fluoride) with previous prophylaxis using prophylactic paste SHINE ${ }^{\circledR}$ of Maquira (2000 ppm of F), the final elements reduction of the average cervico-occlusal and mid-distal diameters were observed in 08 elements and maintenance of the averages in 02 . In addition, 02 white spots remained active, the others inactive, concluding that there was remineralization of white spots with a reduction in diameter and / or activity after 8 therapy sessions with fluoride varnish and guidance on the patient's hygiene and diet.

KEYWORDS: Tooth Decay. Demineralization. Fluoride.

\section{REFERÊNCIAS}

1- Ministério da Saúde (BR). Projeto SB Brasil 2010: Pesquisa Nacional de Saúde Bucal Resultados Principais. Brasília, DF: Ministério da Saúde; 2011.

2- Parisotto TM, Steiner-Oliveira C, De Souza-ESilva CM, Peres RC, Rodrigues LK, Nobre DosSantos M. Assessment of cavitated and active non-cavitated caries lesions 3-to 4-yearold preschool children: a field study. Int J Paediatr Dent. 2012; 22(2): 92-9.

3- Matltz M, Tenuta LMA, Groisman S, Cury JA. " Cariologia: Conceitos básicos, diagnóstico e tratamento. 1ed. São Paulo: Artes Médicas, 2016. 4- Bulgareli. JV, Caldareli PG, Freitas FF, Pereira PG, Rodrigues LP, Zancopé BR. "Revisão de literatura: odontologia preventiva em pacientes ortodônticos - como prevenir e tratar as lesões de mancha branca?" Rev. Multidiscipl Dent. 2020;10 (1):52-8.

5- Atlan A, Denis M, Tirlet G, Attal JP. Orthodontics and enamel spots. Benefits of a minimally invasive approach, guidelines for the orthodontist. J Dentofacial Anom Orthod, 2017; 20(3) 1-19.

6- Bussaneli DG. Control of White Spot Lesion
Adjacent to Orthodontic Bracket with Use of Fluoride Varnish or Chlorhexidine Gel. J Clin Pediatr Dent. 2016; 40(4): 274-80.

7- Geraldo-Martins, V. R., Barbosa, B. G., da Silva, V. L., Gontijo, I. G., \& Nogueira, R. D. Tratamento de lesão de mancha branca com infiltrante resinoso: relato de caso. Rev Odontol Bras Central 2018; 27(83): 252-56.

8- Ferreira. JMS, Aragão AKR, Rosa ADB, Sampaio FC, Menezes VA. Therapeutic effect of two fluoride varnishes on White spot lesions: a randomized clinical trial. Braz Oral Res. 2009; 23(4): 446-51.

9- Silva RM, Ferreira JMS, Silva CDB, Fontes LBC, Granville-Garcial AF, Menezes VA. In vivo Evaluation of therapeutic potencial of fluoride varnishes. Rev Odonto Cienc. 2012; 27(3): 233-37.

10- Lucchese A, Gherlone E. Prevalence of whitespot lesions before and during orthodontic treatment with fixed appliances. Eur. J. Orthod. 2013; 35(5): 664-68.

11- Ogaard, B. "White spot lesions during orthodontic treatment: mechanisms and fluoride preventative aspects." Semin Orthod. 
2008; 14(3): 183-93.

2- Lapenaite E, Lopatiene K, Ragauskaite A. Prevention and treatment of white spot lesions during and after fixed orthodontic treatment: $\mathrm{A}$ systematic literature review. Rev. Stomatologija. 2016; 18(1):3-8.

13- Khalaf K. Factors Affecting the Formation, Severity and Location of White Spot Lesions during Orthodontic Treatment with Fixed Appliances. J Oral Maxillofac Res. 2014; 5(01): 1-10.

14- Poosti M, Ahrari F, Moosavi H, Najjaran H:
The effect of fractional $\mathrm{CO} 2$ laser irradiation on remineralization of enamel white spot lesions. Lasers Med Sci. 2014; 29(4): 1349-55.

15 -Benson, PE, Shah AA, Millett DT, Dyer F, Parkin N, Vine RS. "Fluorides, orthodontics and demineralization: a systematic review." J Orthod. 2005; 32(2): 102-14.

16- Bishara, S, Ostby A. "White spot lesions: formation, prevention, and treatment." Seminars in Orthodontics. 2008; 14(3): 174-82.

17- Tenuta LM, Cury, JA. Fluoride: its role in dentistry. Brazilian Oral Res. 2010; 24(01): 9-17. 